\title{
Modelica library for the systems engineering of railway brakes
}

\author{
Marc Ehret \\ Institute of System Dynamics and Control, German Aerospace Center, Germany, marc.ehret edlr. de
}

\begin{abstract}
This work outlines the role of system simulation for the development process of railway brakes. The principles of systems engineering motivate the use of computer based simulation in order to enhance the understanding of systems and to verify the behavior of systems in early design phases. For this reason, the Modelica library "Virtual Train Brakes" is presented which enables engineers to generate simulation models of railway brakes and to perform system simulations during different phases of the development process. By modeling and simulating the brake systems of a passenger and a freight train, the capability of the library is demonstrated and further investigations are motivated.

Keywords: Railway Brakes, Systems Engineering, MultiLevel Models, Variant Models, Generic Models
\end{abstract}

\section{Introduction}

Railway brakes are complex technical systems that fulfill high requirements concerning safety, availability and reliability. These systems are constructed to be deployed in a difficult operational environment, characterized by large temperature ranges and heavy dynamic loads during long utilization times. Furthermore, a huge amount of kinetic energy needs to be converted during a single brake application which requires that brake discs are able to absorb up to $30 \mathrm{MJ}$ of energy (Breuer, 2006). Beside severe operational conditions, the diversity of railway vehicles and brake types demands to design individual adapted brake systems which leads to a high variety and individuality of the developed systems. This individuality and the corresponding variety of brake systems is in contrast to the comparatively low number of items that is usually delivered (Anton, 2010). In order to reduce technical as well as economical risks the application of systems engineering is indispensable for of the development process of railway brakes.

Systems engineering is an approach that enforces system architects to deduce physical solutions by identifying stakeholders, specifying their requirements and map them by system functions. Thereby, an interdisciplinary and holistic view of the desired system is generated, which is deepened throughout the design process. By this means, mistakes are identified and eliminated in early design phases before costly changes during the implementation are necessary. Due to the increasing complexity and functionality of technical systems arising from the increasing content of electronic and software components, systems engineering has become a wide spread and important procedure in product development (INCOSE, 2015).

In the context of systems engineering, computer based system simulation is a powerful tool since it supports designers to understand the behavior of systems in design stages where an experimental analysis is not feasible. Furthermore, it allows engineers to check their own thinking, to analyze alternatives and capabilities of the system and to communicate their concept to others (INCOSE, 2015) (Mittal et al., 2017). Modelica is a well suitable modeling language to generate models of multi-physical technical systems, such as railway brake systems and to study their physical behavior.

The goal of this work is to develop the concept of a Modelica library which provides an environment for the application of system simulation throughout the entire development process of railway brakes. As shown in Figure 2, the main tasks of the library are:

- support dimensioning

- analyze and optimize system behavior

- support integration

- support system test and commissioning

Initially, this work introduces railway brake systems and the role of systems engineering in the scope of their design. Subsequently, use cases of system simulation during the development process are discussed which are the basis for the structure and implementation of the library with Modelica in Dymola. Varying requirements concerning the accuracy and the computational effort of the models are considered and the generic composition and diverse configuration variants of railway brake systems are respected. After the presentation of the library it is applied to model and simulate the brake systems of a passenger and a freight train. The development of the library is an ongoing work. This paper is primarily meant to motivate its usage and to present the modeling concept. An outlook is given which states the current limits of the library and motivates further investigations. 


\section{Systems engineering of railway brakes}

\subsection{Introduction to railway brakes}

The main functions of railway brake systems are (i) the conversion of kinetic energy in order to reduce the velocity of the train, (ii) to counteract the downhill-slope force in order to keep the velocity of the train and (iii) to prevent a stationary train of moving due to gradients or other external forces, for instance caused by wind (Knorr-Bremse, 2003).

The basic safety requirements demand that all types of railway vehicles have an automatic, continuous and inexhaustible brake system. This means that the brake systems of all cars of a railway vehicle are controlled by a through signal line (continuity) and that in case of an error in this signal line caused by leakage or cutoff, each of the cars of the vehicle stops automatically. Inexhaustibility requires that the performance of the brake system is still available, although there have been repeated brake applications before (DIN EN 14198, 2005) (DIN EN 15734-1, 2013). The brake distance in case of emergency braking is the major performance requirement brake systems have to meet. The maximum brake distances of locomotives and passenger trains required by (Commission Regulation (EU) No 1302/2014, 2014) is shown in Table 1 relating to different velocities. Additionally, the mean value of the corresponding deceleration is given assuming a constant deceleration. This value does not include the delay and response time of the brakes as well as the velocity-dependent adhesion between wheel and rail limiting the maximum feasible deceleration described in chapter 4.2.4.5.2 and 4.2.4.6.1 in (Commission Regulation (EU) No 1302/2014, 2014).

Table 1. Brake distance requirements for emergency braking of locomotives and passenger trains relating to different initial velocities according to (Commission Regulation (EU) No 1302/2014, 2014)

\begin{tabular}{ccc}
\hline $\begin{array}{c}\text { Initial } \\
\text { velocity }\left[\frac{\mathrm{km}}{\mathrm{h}}\right]\end{array}$ & $\begin{array}{c}\text { Required } \\
\text { brake distance }[\mathrm{m}]\end{array}$ & $\begin{array}{c}\text { Mean } \\
\text { deceleration }\left[\frac{\mathrm{m}}{\mathrm{s}^{2}}\right]\end{array}$ \\
\hline 350 & 5360 & 0.88 \\
300 & 3650 & 0.95 \\
250 & 2430 & 0.99 \\
200 & 1500 & 1.03 \\
\hline
\end{tabular}

In order to fulfill these requirements and functions railway vehicles are equipped with different types of brake systems, shown in Figure 1, depending on the type of vehicle (passenger cars, freight cars, locomotives) and its operating modes (service brake, emergency brake, parking brake, holding brake).

Due to its high level of safety all railway vehicles are at least equipped with frictional brake systems, such as

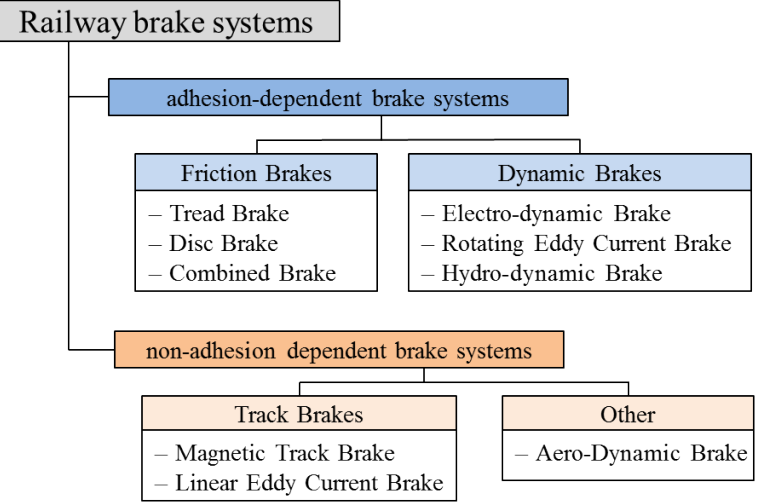

Figure 1. Classification of railway brakes according to (KnorrBremse, 2003)

tread and disc brakes, which are mostly activated by compressed air except for trams which use hydraulic media due to the limited available space in these vehicles. Additional brake systems, e.g., electro-dynamic brakes in electrically driven vehicles and hydro-dynamic brakes in diesel-hydraulic vehicles, are applied to serve as service brakes and to support the frictional brakes in order to minimize wear and thus extend the technical lifetime of brake systems. Non-adhesion dependent systems, for instance track-brakes, are not limited by the maximum transferable force between rail and wheel. They are deployed to minimize the braking distance in case of an emergency. The faultless cooperation and redundancy of the diverse subsystems is an important aspect for the safe, secure and reliable operation of trains.

\subsection{Systems engineering}

Depending on the type of system there are different process models which determine the design procedure of systems. Haberfellner and Daenzer differentiate between plan-driven methods, such as the "V-Model" or the "Waterfall-Model" and agile methods, such as "Scrum" or the "Spiral-Model" (Haberfellner and Daenzer, 2002). The latter methods are commonly used for software engineering in which flexible and less sequential frameworks are preferred due to the dynamic and changing environment throughout the development process. In contrast, plan-driven methods are characterized by fixed steps and defined sequences during the development process. This is essential for the design of large multi-physical systems which are subject to high requirements regarding safety and reliability, such as railway brake systems.

The most common plan driven method is the "VModel", as shown in Figure 2. The basic idea is to follow a structured top-down development process. At first the stakeholders are identified, who are the source of the system requirements from which in turn the system specifications are deduced (Haberfellner and Daenzer, 2002) (INCOSE, 2015). During the top-down development process the specifications of the system are disassembled into 


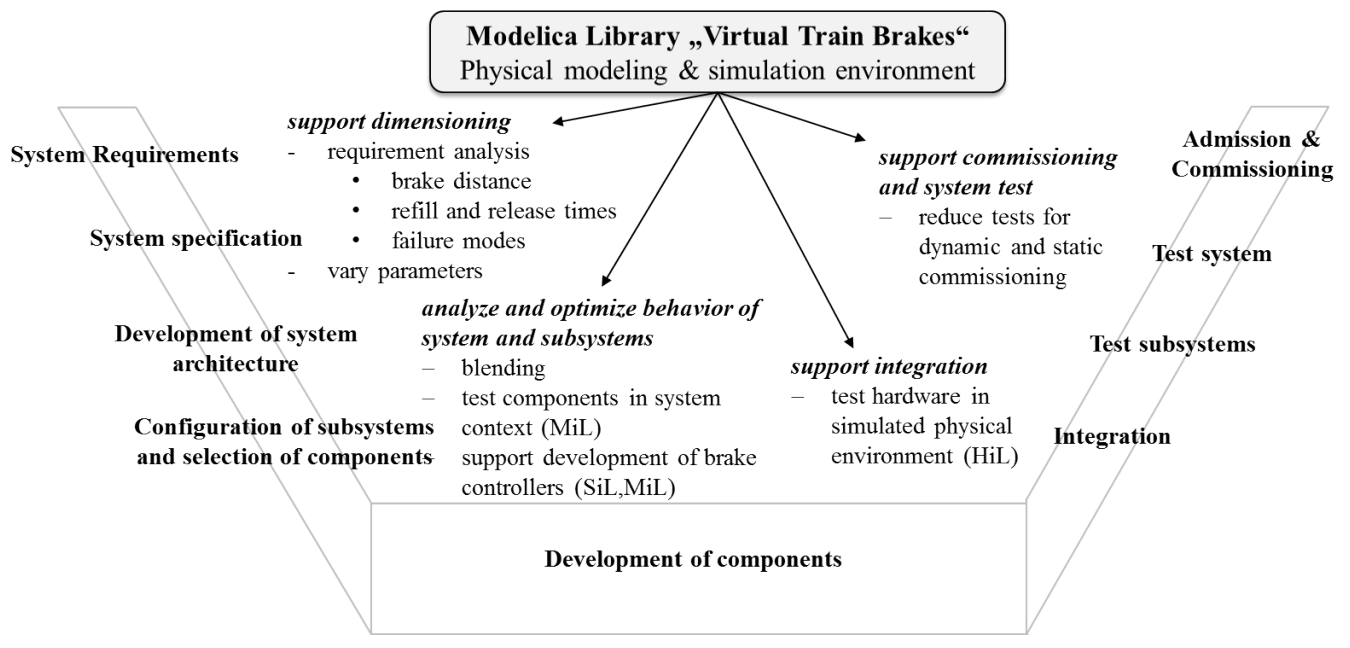

Figure 2. "V-Model" as development process of railway brake systems with use cases for system simulation

sub-specifications of subsystems until the smallest entity is reached. In each stage the physical architecture, system elements and interfaces are derived from the specifications using context and functional analysis. During the bottomup implementation and integration phase the elements and subsystems are built and verified, if they fulfill the defined specifications. Finally, this leads to the integration, verification and validation of the entire system in its operational environment.

The development of railway brakes is a typical case of application engineering, since brake systems consist of standard components provided by an existent product asset, such as brake cylinders, typical valves and preassembled subsystems like integrated modules for brake control (André et al., 2017). Application engineering is a particular type of systems engineering in which the elementary components and subsystems are predefined parts of a component portfolio. Many technical systems are designed by reusing predefined and for the system characteristic components which are selected, configured and interconnected.

\subsection{Simulation of railway brakes}

To analyze the time dependent behavior of railway brakes physical models can be applied, which are represented by mathematical equations deduced from natural laws. The object-oriented and component-based modeling approach of Modelica is well suited to develop these physical models and is therefore used in this work. The goal of the Modelica library "Virtual Train Brakes" is to provide physical models for system simulation throughout the entire systems engineering process of railway brakes shown in Figure 2.

As stated in (Anton, 2010), the ambivalence of cost for design and quality of the designed system in scope of the tender process of railway brakes strongly motivates the use of system simulation. During the tender process system engineers design the rough architecture of brake systems. In this phase the type of applied brake systems as well as the number of brakes and their dimensions are defined depending on the specification of the customer. Modeling and simulating the designed architectures allows to gather useful information regarding the system behavior, such as the effects of refill and release times or failure modes on the braking distance. Furthermore, the sensitivity and impact of varying system parameters, e.g., the size of reservoirs and diameters of pipes, can be analyzed. Thus, system simulations help to avoid oversizing of systems and to submit competitive offers during the tender process.

As mentioned in 2.1 railway vehicles are usually equipped with several types of brake systems. To achieve the desired deceleration while minimizing wear a complex brake management is necessary. The so called blending defines the contribution of the different brake systems relating to the current operating mode. System simulation facilitates the analysis and review of the individually designed blending concepts long before commissioning. Furthermore, it allows designers to apply numerical optimization in order to find the ideal blending concepts.

Another application of system models is to simulate the physical environment of components or subsystems. This enables system designers to study and review the behavior of components or subsystems in the context of their environment. During the development of electronic devices, such as control units, a model representing the basic control algorithm ("Model in the Loop": MiL), the implemented software ("Software in the Loop": SiL) and finally the hardware of the unit ("Hardware in the Loop": HiL) are designed and verified by integrating them into a simulated physical environment as described in (Tischer and Widmann, 2012). Depending on the integrated object different requirements concerning the computation rate, model interfaces and the accuracy of the simulation need to be considered. The application of HiL-testing for the development of railway brake systems is demonstrated in (Pugi et al., 2006), (Kang et al., 2009) and (Lee and Kang, 2015). 
Finally, virtual testing with validated system models allows system engineers to reduce the efforts of experimental testing which is directly linked to time and costs for implementation, integration and commissioning. The main intention of virtual testing is not to replace experimental tests, but to narrow the quantity of tests which are necessary to adjust the detailed setting of components, e.g., valves and nozzles, in order to harmonize and verify the entire system behavior.

To provide physical models throughout the entire development process a model library is required which bases on the component and system portfolio of railway brakes. Furthermore, the presented scenarios for system simulation demand models which are customizable with respect to the level of detail, accuracy and computational effort depending on the scope of simulation.

\section{Modelica library "Virtual Train Brakes"}

The Modelica library "Virtual Train Brakes" is an approach to realize the desired simulation environment. Initially, the structure and modeling concepts are introduced followed by the implementation in Modelica.

\subsection{Library structure and modeling concepts}

The structure of the library is pictured in Figure 3. The foundation is the component library which contains multiphysical models of typical technical components analog to the product asset of railway brakes, for instance distributors valves, pressure transformers or brake cylinders. These technical components consist of core elements from different physical domains, such as nozzles and volumes in case of pneumatic components. In this work core elements from the Modelica Standard Library and from the Pneumatics Library developed by Modelon, described in (Modelon AB, 2010) and (Beater, 2007), are applied. To fulfill the requirements of adaptable accuracy and computation rate the models of the technical components are implemented as multi-level models. This means that different levels of detail of a component are depicted by selectable level-models which share the same physical interfaces but differ in their modeling content.

The technical components provide the elements for the system library. This library contains generic models of systems and subsystems of railway brakes what eases the generation of large models. The pre-assembled system models result from the definition and separation of brake systems into subsystems, including boundaries and interfaces analog to the top-down development process of the "V-Model". In case of the system "train", which is exemplarily subdivided in Figure 4, the system model in the highest layer (Layer 1) consists of the subsystems "car". This contains the subsystems "brake panel", "bogie pneumatic" and "bogie mechanic" which in turn include interconnected technical components, e.g., distributor valves and the pressure transformers. In this generic concept

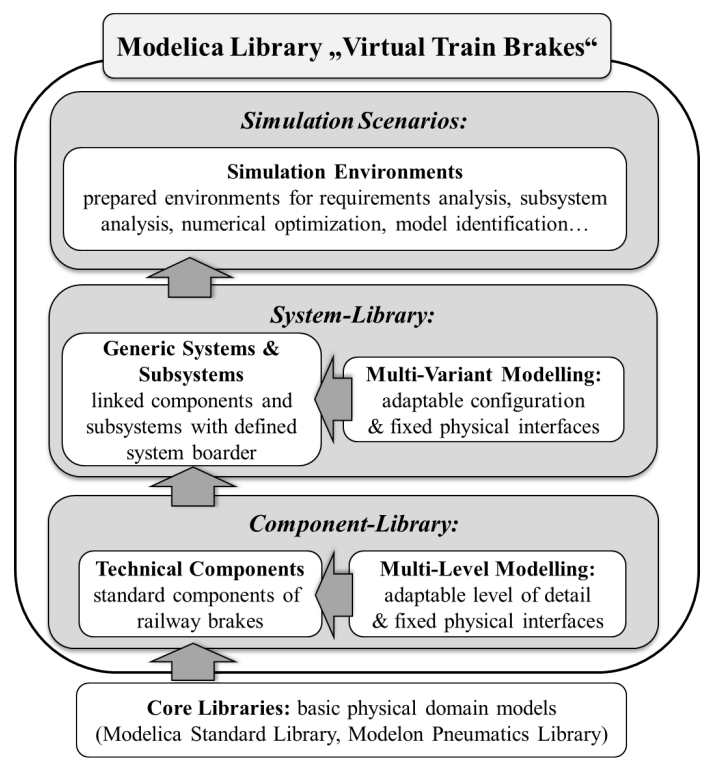

Figure 3. Structure of the library "Virtual Train Brakes"

components can be part of any subsystem in any layer.

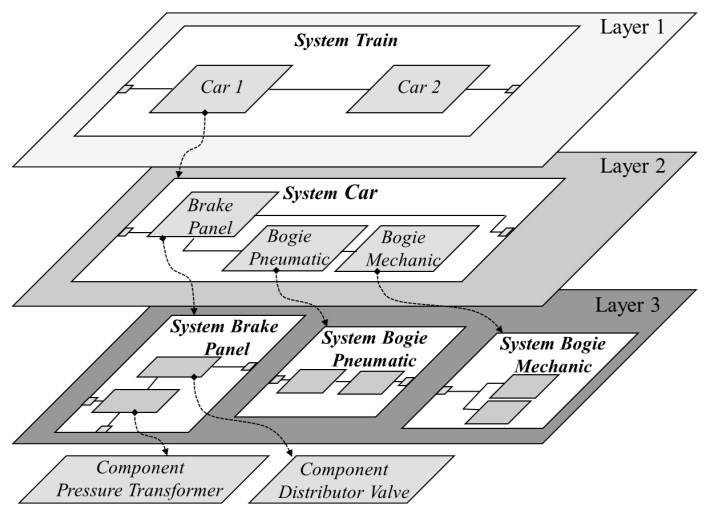

Figure 4. Exemplary scheme of the generic system "train"

Railway brake systems are individually designed depending on the varying specifications and on the vehicle they are integrated into. This leads to a broad diversity of designed systems and it is unfeasible to provide generic models for all them. Nevertheless, particular subsystems are combined to modular platforms for specific product families of which certain variants exist (André et al., 2017). By identifying and modeling these standard variants it is possible to ease the model generation for a large amount of railway brake systems. For this reason, the generic models of the system library are implemented as multi-variant models differing in their configuration but sharing the same physical interfaces, as exemplarily shown in Figure 5 for the subsystem "brake panel". The restriction of having the same interfaces is indispensable. Otherwise the integration of a configurable subsystem into a higher layer could fail due to violation of the interconnections between a configured subsystem and the elements in its environment. Furthermore, the complexity of the generic models is limited and an extensive configu- 
ration management is avoided.

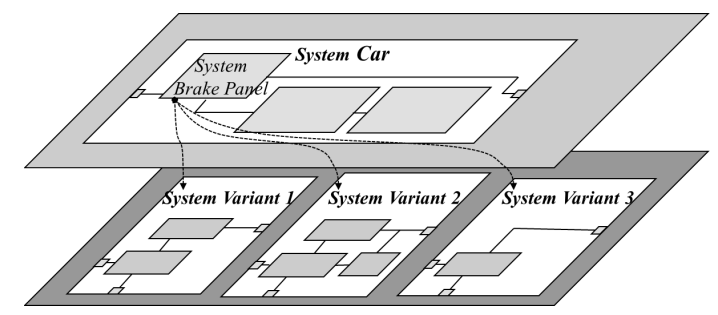

Figure 5. Exemplary scheme of variant modeling in a generic system structure

\subsection{Implementation of the library in Modelica}

According to the presented structure the Modelica library is subdivided into a component library and a systems library in which the models are stored. In the following, the implementation of component models and system models is described.

\subsubsection{Modeling multi-level components}

The implementation of a technical component is described by using the example of the component "Brake Cylinder". The corresponding component package contains a so-called container model in which two instances of the models "BrakeCylinderLevel_1" and "BrakeCylinderLevel_2" exist in parallel, as shown in Figure 6 and suggested by (Kuhn et al., 2008). They represent two different modeling approaches which basically differ in their level of detail. Both level-models are connected to the ports of the container model where they are implemented by using conditional statements. This means that the models are not considered when translating the container model, if the corresponding conditional statement is false. The activation of the desired model is controlled by an Integer parameter called "Level" which activates "brakeCylinderLevel_1" in case of a value equal to 1 and "brakeCylinderLevel_2" for a value equal to 2 . Thus, the selection of the desired model is controlled by a single parameter and does not have to be carried out manually as it would be done in case of replaceable models (Kuhn et al., 2008).

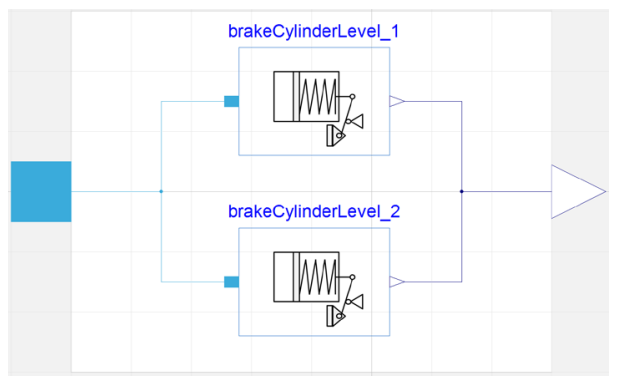

Figure 6. Container model of the component "Brake Cylinder" with two conditionally implemented level models

The container model includes an instance of the record "ParameterBrakeCylinder". This record contains the parameter "Level" and instances of individual records of the level-models "ParameterBrakeCylinder_Level_1" and "ParameterBrakeCylinder Level 2" leading to a clear separation of the model-dependent parameter sets.

To ensure that the container model and the level-models are compatible with each other they are inherent classes of the class "BrakeCylinderTemplate". This model defines the interfaces and the icon of the component model. Thereby, the component models represent the same ports, regardless of the selected level-model. This is important for a clean integration of component models into system models.

Additionally, the component package provides a simulation environment "SimulateBrakeCylinder" which integrates the model into a testing environment. This is helpful for testing newly developed models and serves as simulation example when applying the component, respectively. Furthermore, it is possible to compare simulation results with experimentally measured data for the verification of the behavior of the model, as shown in 4.1.5. In this case it is important to ensure that the simulation environment represents the initial and boundary conditions which existed during the corresponding experiment.

Figure 7 and 8 show the content of the two level-models of a brake cylinder which convert pneumatic pressure into a clamping force. In model "brakeCylinderLevel_1" the pneumatic input port on the left hand side is connected to a constant pneumatic volume. The pressure in this volume is used to calculate the resulting clamping force depending on parameters, e.g., piston diameters and efficiency by using a simple formula. In contrast, "brakeCylinderLevel_2" uses mechanical and pneumatic components, such as a pneumatic cylinder, a counteracting spring, the brake rigging and a gap element to model the contact between disc and brake pad. This represents a more detailed model of the component taking further physical effects into account, for instance friction, contact effects and pressure dependent volumes.

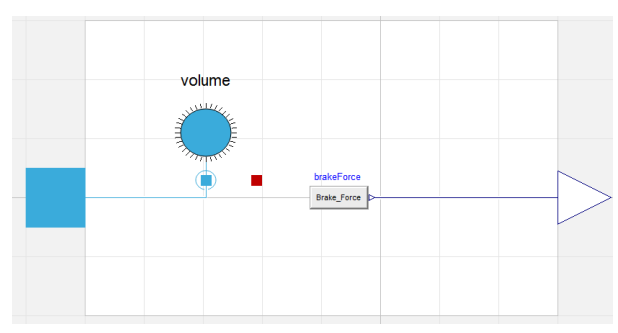

Figure 7. Model content of "BrakeCylinderLevel 1" with volume model from the Modelon Pneumatic Library

Figure 9 shows the impact of the two different modeling approaches on simulation results by comparing the calculated clamping force in case of a pressure gain in the cylinder. The simulation results of the model "brakeCylinderLevel_2" contain transient effects at the beginning. They are caused by friction in the pneumatic cylinder of and the contact of pad and disc. These effects might be of interest in case of a detailed investigation, for example the 


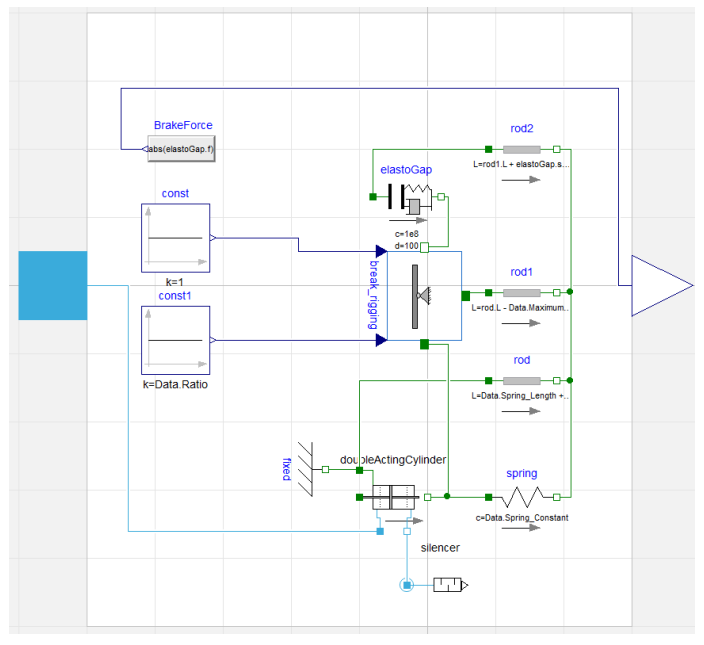

Figure 8. Model content of "BrakeCylinderLevel 2" with cylinder model of the Modelon Pneumatic Library

behavioral analysis of the brake cylinder during the application of the wheel slide protection. Model "BrakeCylinderLevel_1" neglects these effects, but provides the same stationary clamping force. This is sufficient for the analysis of the entire brake system, for instance in case of the estimation of the brake distance.

A comparison of the computation time in Figure 10 shows that the simulation of model "BrakeCylinderLevel_1" (2 states, 0.03 seconds) is 5 times faster than the simulation of model "BrakeCylinderLevel_2" (9 states, 0.16 seconds). Especially the computation of the transient oscillations after 0.2 demands a large computational effort. The bigger the system of interest, the stronger the entire computation rate is influenced by the computational effort for single components. This example emphasizes the need for different model approaches due to the varying scope of simulations described Figure 2.

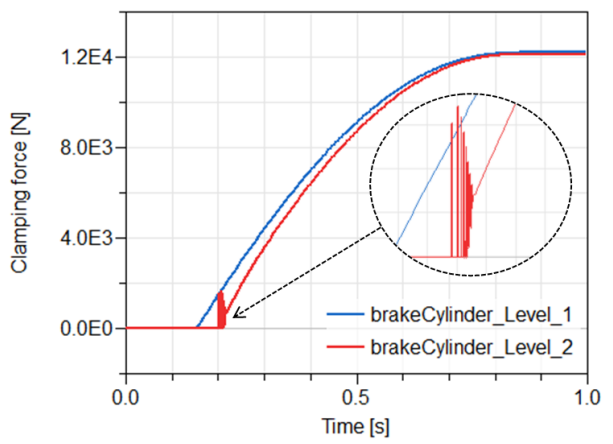

Figure 9. Comparison of simulation results of the level-models "brakeCylinder_Level_1" and "brakeCylinder_Level_2" with zoomed transient effects

\subsubsection{Modeling multi-variant and generic systems}

To model multi-variant system models, container models are applied analog to the modeling approach of multi-level components described in 3.2.1. The use of container mod-

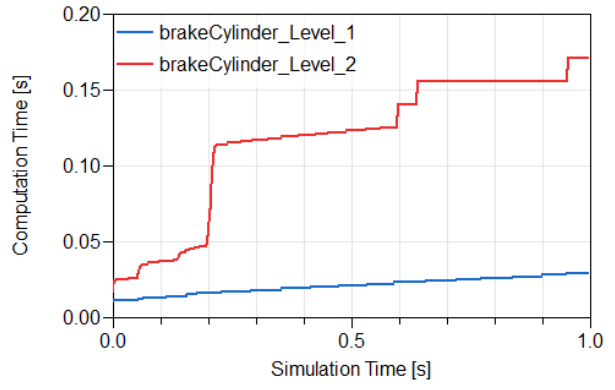

Figure 10. Comparison of computation time of the models "brakeCylinder_Level_1" and "brakeCylinder_Level_2"

els in place of replaceable models enables the user to configure system models by supplying parameters instead of choosing the desired variant manually. The essential difference between the models of systems and components is that the container model a system includes variant-models. They represent different configurations of a system instead of different modeling approaches concerning the level of detail. Thus, the structure of a system package is analog to the component package. Since the parameter sets of different system configurations are mostly the same, there are no individual records for each of the variant-models in contrast to the level-models of components.

To cover further configuration types of a system, integrated components and subsystems are implemented by using conditional statements. These submodels can be deactivated, if they do not appear in a particular system configuration. In this case it is important to ensure that singularities due to missing states are avoided.

By offering variant-models and the possibility to add or remove particular submodels in these variant-models a broad diversity of system configurations can be represented by using a single container model.

Figure 11 shows the GUI of the record "ParameterBrakePanel" of the system "Brake Panel". To select the desired system variant the integer variable "Variant" is added. Furthermore, boolean parameters can be found, such as "DV_existent" or "EPC_existent" which activate or deactivate integrated submodels (DV: Distributor Valve, EPC: Electro pneumatic Control).

Beside configuration parameters this record contains records of the integrated sub-models, as shown in Figure 11. The parameter "Level_Submodel" serves as default value for the parameter "Level" in the records of the underlying components. Thus, their level of detail may be controlled globally by a single value. If a sub-model is a generic system itself, the selection of the corresponding table is leading to a record with the same structure. Thereby, the parameters of a generic system are hierarchically ordered analog to the generic system structure. Finally, this approach leads to a single record that contains all parameters of all integrated components and subsystems to parameterize and configure the entire generic system model. 


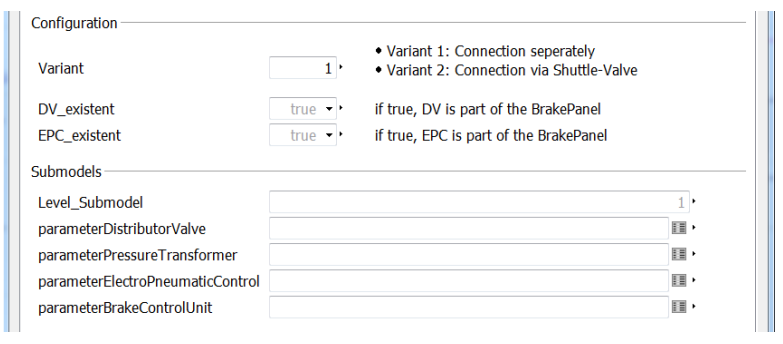

Figure 11. GUI of the record "ParameterBrakePanel"

\section{Application scenarios}

In the following the library "Virtual Train Brakes" is used to model the railway brake systems of a passenger and a freight train. By performing system simulations according to use cases depicted in Figure 2 exemplary data is generated which serves as basis for the behavioral analysis of the brake systems.

\subsection{Modeling and simulating a passenger train}

\subsubsection{Model generation}

Figure 12 presents a generic model of the frictional pneumatic and electro-pneumatic brake system of a passenger train with three railcars. The train is assembled of the submodels "railcar" and "train control" which are preassembeled generic models of the system library. The cars are connected with pneumatic lines supplying energy and pressure signals to activate the friction brakes and an additional bus line containing electronic brakes signal. To determine the brake distance and velocity of the train, position and speed sensors are connected with mechanical flanges of the railcars.

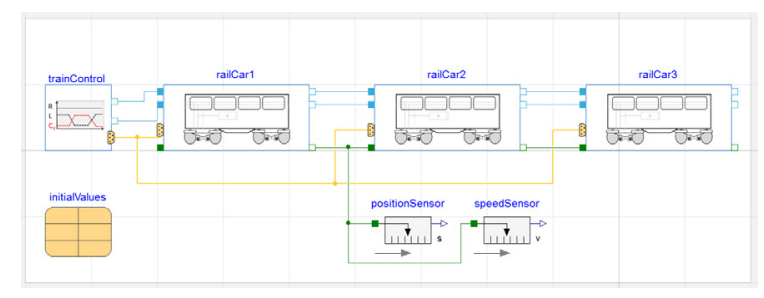

Figure 12. Model of a passenger train with three railcars

Figure 13 depicts the selected variant-model of the first railcar. It contains the subsystems "brake panel", "bogie pneumatic" and "bogie mechanic" as well as components, such as pipes, reservoirs and the pneumatic suspension. The subsystem "bogie mechanic" includes models which calculate the resulting brake forces that decelerate the mass of the train. In these models the friction forces between disc and brake pad are calculated using the clamping forces of the brake cylinders and the corresponding frictional coefficient. These friction forces in turn yield the braking torque acting on the axle depending on the friction radius. The translational braking force is calculated by considering the slip in the contact of wheel and rail resulting from the difference of the rotational ve- locity of the braked axle and the velocity of the train.

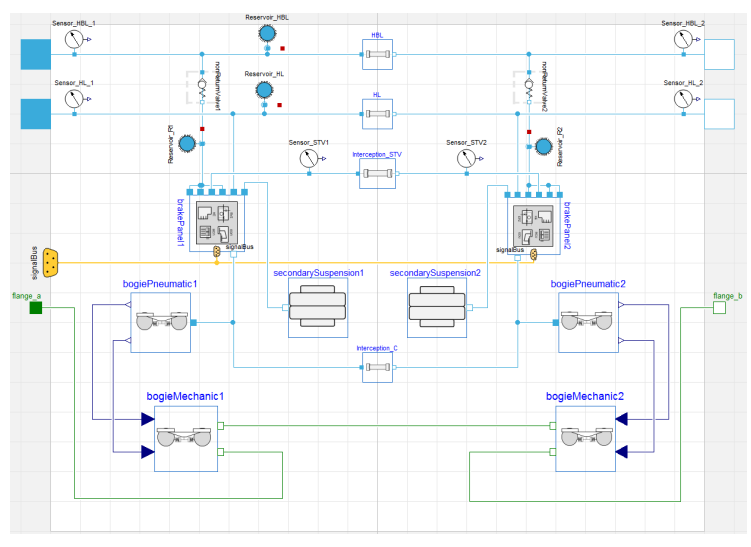

Figure 13. Variant-model of railcar 1

\subsubsection{Requirements Analysis}

In the train model all bogie masses are connected by flanges leading to a single mass model. This is sufficient to estimate the brake distance for different operating conditions and modes. This kind of analysis is an important step during the dimensioning of the brake system, since the brake distance is the major requirement to be met. The library "Virtual Train Brakes" includes the function "EvaluateBrakingDistanceMatrix" which executes automated system simulations with varying initial values, e.g., the velocity of the train, and parameters, for example the radius of wheels or the axle load.

The function is used to simulate an emergency brake application of the passenger train for several cases differing in the initial velocity and the radius of the wheel. The simulation results of the brake distance over time are depicted in Figure 14. Additionally, the function generates a text document containing the values of the calculated brake distance. This overview enhances the verification of requirements based on the simulation results, as shown in Figure 15. It is shown that the effective wheel radius, which might change over the life time of a train due to wear, has a significant influence on the brake distance. This influence becomes more severe with increasing initial velocity.

\subsubsection{Analysis of Refill Time}

Beside estimating the brake distance the presented simulations allow to analyze the states and the related behavior of the system. Figure 16 shows the first 3 seconds of the precontrol pressure and the resulting cylinder pressure $(\mathrm{C}$ pressure) for the run $v 0=44.4 \mathrm{~m} / \mathrm{s}$ and $\mathrm{R}=0.385 \mathrm{~m}$. The refill time of the C-pressure is directly correlated to the resulting brake distance, since it determines the moment when the braking torque is fully available. It is mainly influenced by the volumes and pneumatic resistances of valves and pipes that provide the brake cylinders with air. In this case the refill time takes about 1.25 seconds, as shown in Figure 16. 


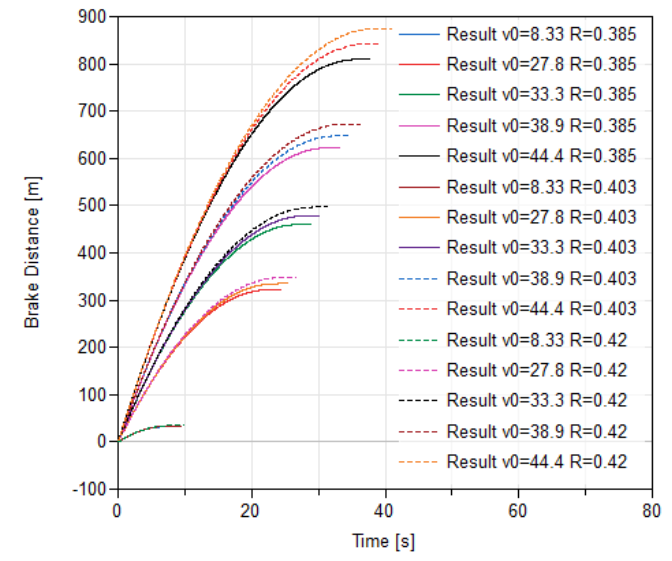

Figure 14. Results of simulations of the generic passenger train model with initial velocity $\mathrm{v} 0$ and radius $\mathrm{R}$ of the wheel

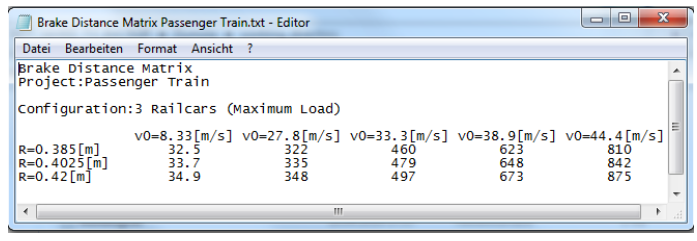

Figure 15. Final brake distances estimated by simulations

\subsubsection{Worst-case analysis}

The use of numerical optimization enables to perform a worst-case analysis. This type of analysis allows to evaluate the limiting parameters of the brake system, which is very useful during the phases of dimensioning and optimization. An exemplary application is the analysis of the friction coefficient in the contact between disc and brake pad. This parameter strongly influences the brake distance and is dependent on the conditions in the contact and the rotational speed of the disc (Knorr-Bremse, 2003).

By applying the optimization library presented in (Pfeiffer, 2012) in conjunction with the system model of the brake a numerical optimization is performed. The goal is to identify the minimum mean value of the friction coefficient that does not violate the brake distance requirement of 950 meters for an initial velocity of $160 \mathrm{~km} / \mathrm{h}$. The start value of the friction coefficient is 0.365 and the maximum range is $[0.1,0.5]$, respectively. The corresponding flow chart is depicted in Figure 17. By automatically tuning the value of the friction coefficient within the defined interval and performing simulations of the adapted model the optimization seeks for the limiting value which leads to a violation of the required brake distance. Figure 17 shows the table of the log-file containing the final results of the optimization. The optimization detects a minimum mean value for the friction coefficient of 0.335 . This value causes a brake distance of 950 meters with a deviation of 0.559 meters which is the value of the minimized criteria $\left|s-s_{R}\right|$ within the interval defined by $\varepsilon$. The design of the brake pad and the disc needs to ensure that the components are able to fulfill the detected limit.

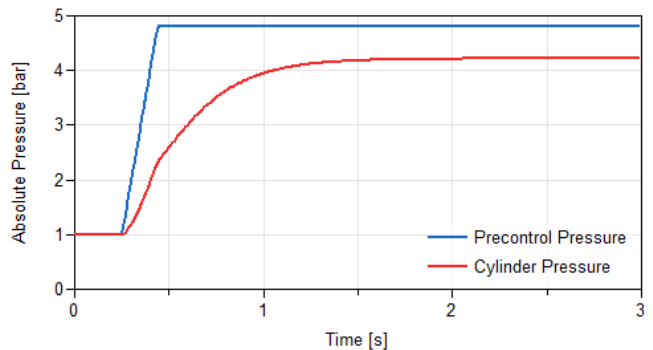

Figure 16. Results of precontrol pressure and resulting cylinder pressure of run $\mathrm{v} 0=44.4 \mathrm{~m} / \mathrm{s}$ and $\mathrm{R}=0.385 \mathrm{~m}$
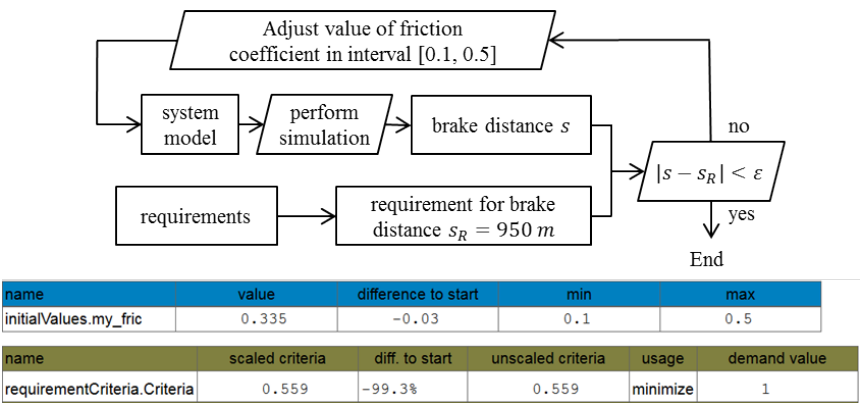

Figure 17. Flow chart of worst-case analysis and final result

\subsubsection{Model Identification}

Another application of numerical optimization in terms of system simulation is the identification of sensitive model parameters for the validation of models by measurements. The validation of component models is important to generate reliable simulation results of an assembled system.

The component "pressure transformer" influences the fill- and release times of the brake cylinders and thus the brake distance of the train. This relay valve provides the brake cylinders with compressed air from a reservoir and deaerates the cylinders in order to release the brakes (Knorr-Bremse, 2003). The sensitive parameters of the corresponding component model are identified by applying a numerical optimization with data from an experimental component test. The corresponding level-model_1 basically contains two nozzles which determine the dynamic behavior of the component in case of venting and deaerating. The sonic conductance is the sensitive parameter of a pneumatic resistance influencing the mass flow at a particular pressure ratio (Beater, 2007). By varying the sonic conductance of these nozzles in scope of a numerical optimization, the model is tuned to minimize the integrated squared deviation of measurement and simulation results. The default value of the sonic conductance of the nozzles is $10^{-7}\left[\mathrm{~m}^{3} / \mathrm{sPa}\right]$ and the boundaries for the optimization are set to $10^{-6}$ and $10^{-8}$. The optimization yields a value of $5.7 \cdot 10^{-7}$ for the sonic conductance of the venting nozzle and $1.9 \cdot 10^{-7}$ for the nozzle controlling the deaeration. The results of measurement and simulation in case of a pressure gain caused by opening the venting nozzle are depicted in 18 . In this figure the 
model with default values for the sonic conductance and the optimized model are compared with the measurement. It shows that the time dependent behavior is well reproduced by the optimized model except for transient effects in the beginning of the measurement. To represent these effects a more detailed model is necessary.

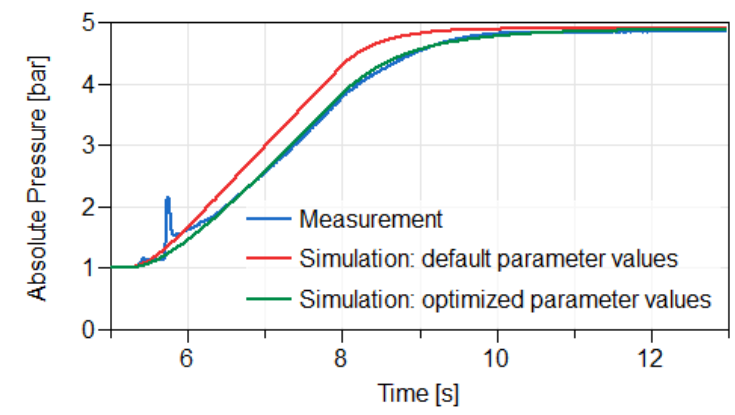

Figure 18. Comparison of measured and simulated C-pressure of distributor valve

\subsection{Modeling and simulating a freight train}

In another example the brake system of a freight train is modeled and simulated. Freight trains consist of a large number of identical cars connected by coupling elements and a long pneumatic line. Pneumatic effects due to the large volume and the length of the line strongly influence the performance of the system. Their investigation requires large experimental effort which is reduced by using system simulation.

In order to ease the model generation of these trains the library offers a model called "generic train". This model contains a front car connected to a vector of generic car models "railcar i[]". The corresponding connections are implemented by using a for-loop in the equation section and yield a model with a selectable number of car in series.

Figure 19 shows simulation results of the deaeration of the continuous brake pipe (BP) in case of a brake application in a freight train with 50 cars. The brake pipe is the through pneumatic signal line of freight trains which causes the aeration of the brake cylinders with compressed air from a reservoir, if the pressure in the line drops below a particular value ${ }^{1}$. Comparing the pressure of the brake pipe in the first car $\left(\mathrm{BP}_{-} 1\right)$ with the last car (BP_50) one can easily observe that the pressure drop in cars in the front is significantly faster than those in the rear part of the train. This dynamic behavior is caused by the limited propagation velocity of the pressure drop as well as the large volume of the long pneumatic line and leads to a strong delay of the pressurization of the brake cylinders at the end of the train. This is shown in Figure 19 by the comparison of the cylinder pressure in first car $\left(C_{-} 1\right)$ and in the last car (C_50). Due to the delay high longitudinal forces might occur which in turn lead to undesirable

\footnotetext{
${ }^{1}$ This is the principle of a indirect pneumatic brake system as described in (Breuer, 2006) and (Knorr-Bremse, 2003)
}

oscillations of the train set.

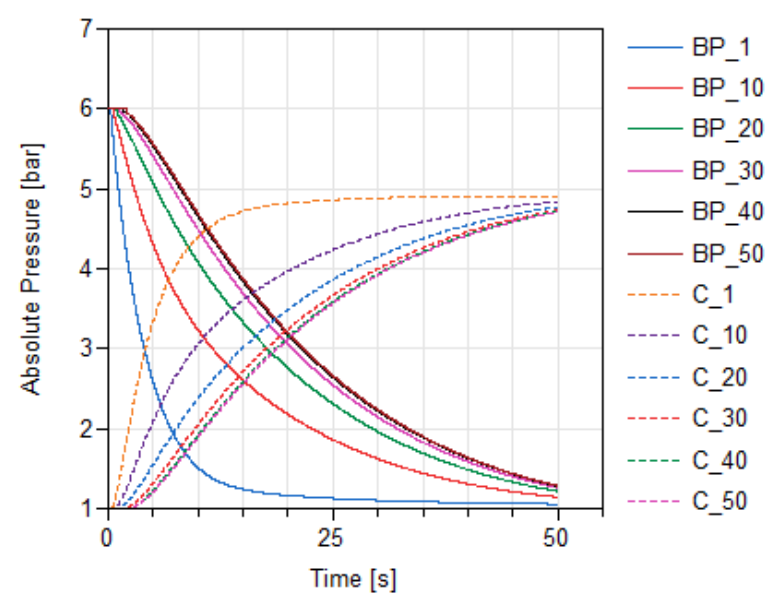

Figure 19. Simulation results of pressure in brake pipes (BP_i) and brake cylinders (C_i) of car $\mathrm{i}=1,10,20,30,40$ and 50 in freight train

\section{Conclusion and Outlook}

By the application of the library "Virtual Train Brakes" for the analysis of the brake systems of a passenger train and a freight train the potential of system simulation during the design and verification of railway brakes is presented. The possibility to select models of typical technical components and to use generic models for varying system configurations drastically ease the generation of models of railway brakes. Simulations of brake application scenarios generate useful knowledge of the system behavior. This can be used to verify a system against its requirements and to analyze the states of the system yielding the resulting behavior. Furthermore, the application of numerical optimization allows to study the system behavior with respect to sensitive parameters and to identify parameters for the validation of models.

Nevertheless, the development of the library is an ongoing process and the presented results motivate further investigations. The implemented models of the library yet focus on simulations of the pneumatic actuation of frictional brakes. The next step is to add further brake types as listed in Figure 1. This means to develop and integrate models of dynamic brakes and non-adhesion dependent brakes in order to simulate and analyze further brake application modes and blending scenarios. Another expansion of the library is the consideration of vehicle dynamics during brake applications. In the current version of the library the railway vehicle is modeled as a rigid mass on a straight track. This is sufficient to estimate the brake distance. However, to analyze the longitudinal dynamic behavior of the vehicle caused by brake applications more detailed models are necessary. These models might include curved tracks, inclination and downhill slope forces as described in (Spiryagin et al., 2014). Beside the development and integration of more detailed dynamic mod- 
els it is also possible to combine brake system models of the presented library with railway vehicle models of other libraries, such as models presented in (Heckmann and Grether, 2017).

Concerning frictional brakes the thermal behavior of brake discs needs to be taken into account during system simulations. The goal is to estimate the temperature of the brake disc during brake applications which influences wear and the friction between disc and pad as described in (Ostermeyer, 2001). This demands to estimate the energy absorbed by the discs and to consider the heat transfer due to conduction, radiation and convection. Additionally, more attention needs to be payed to the modeling of the instantaneous friction coefficient between disc and pad which is influenced by the contacting materials, their temperature, relative speed and normal forces in the contact area. Parameters of models describing these correlations can be estimated from dynamometer measurements as shown in (Lee and Kang, 2015).

Finally, the use of system simulation during the development process demands the validation of entire system models. Therefore, it is necessary to obtain data from train tests, such as integration tests during static and dynamic commissioning, and to perform analog system simulations to verify the system models and ensure that the simulation results are reliable.

\section{Acknowledgments}

The presented results arise from an ongoing scientific cooperation of the Institute of System Dynamics and Control of the "German Aerospace Center" and the "KnorrBremse Systeme für Schienenfahrzeuge GmbH". The resulting Modelica library is a prototype for an in-house tool and is not supposed to be publicly available. I would like to thank all colleagues from Knorr-Bremse for the strong support during the common project.

\section{References}

Jean-Marc André, Hartmut Mann, and Jörg-Johannes Wach. Integrierte Bremssteuerungsmodule zur schnellen Umsetzung sehr unterschiedlicher und komplexer Anforderungen. ZEVrail, 141(10):397-401, October 2017.

Thomas H.F. Anton. Entwicklungs- und Einführungsmethodik für das Projektierungswerkzeug Pneumatiksimulation. PhD thesis, Technische Universität München, 2010.

Peter Beater. Pneumatic Drives. Springer-Verlag BerlinHeidelberg, 2007. ISBN 10 3-540-69470-6.

Bert Breuer. Bremsenhandbuch. ATZ / MTZ-Fachbuch. Vieweg+Teubner Verlag, Wiesbaden, 2006. ISBN 9783663094418.

Commission Regulation (EU) No 1302/2014. Concerning a technical specification for interoperability relating to the 'rolling stock - locomotives and passenger rolling stock' subsystem of the rail system in the European union, November 2014.
Reinhard Haberfellner and Walter F. Daenzer, editors. Systems Engineering. Verl. Industrielle Organisation, Zürich, 11th edition, 2002. ISBN 385743998X.

Andreas Heckmann and Gustav Grether. The DLR RailwayDynamics Library: the Crosswind Stability Problem. In Proceedings of the 12th International Modelica Conference, number 132, pages 623-631, 2017.

INCOSE. Systems Engineering Handbook. Wiley, 4th edition, 2015.

Chul-Goo Kang, Ho-Yeon Kim, Min-Soo Kim, and ByeongChoon Goo. Real-time simulations of a railroad brake system using a dspace board. In 2009 ICCAS-SICE, pages 40734078, Aug 2009.

Knorr-Bremse. Grundlagen der Bremstechnik. Knorr-Bremse Systeme für Schienenfahrzeuge GmbH, 2003.

Martin Kuhn, Martin Otter, and Loic Raulin. A multi level approach for aircraft electrical systems design. In Proceedings of the 6th International Modelica Conference, Germany, Bielefeld, March 3-4, 2008, March 2008.

Nam-Jin Lee and Chul-Goo Kang. The effect of a variable disc pad friction coefficient for the mechanical brake system of a railway vehicle. PLoS ONE, 10(8):e0135459, July 2015. ISSN 1932-6203.

DIN EN 14198. Railway applications - Braking - Requirements for the brake system of trains hauled by locomotives, March 2005.

DIN EN 15734-1. Railway applications - Braking systems of high speed trains - Part 1: Requirements and definitions, September 2013.

Modelon AB. Modeling of Pneumatic Systems - Tutorial for the Pneumatics Library V 1.3/1.5.2, 2010.

Saurabh Mittal, Umut Durak, and Tuncer Ören. Guide to Simlation-Based Disciplines: Advancing our Computational Future. Simulation Foundations, Methods and Applications. Springer, 2017.

G. P. Ostermeyer. Friction and wear of brake systems. Forschung im Ingenieurwesen, 66(6):267-272, Oct 2001.

Andreas Pfeiffer. Optimization library for interactive multicriteria optimization tasks. In Proceedings of the 9th International Modelica Conference, Munich, Germany, September 03-05, 2012, pages 669-679, 2012.

L. Pugi, M. Malvezzi, A. Tarasconi, A. Palazzolo, G. Cocci, and M. Violani. HIL simulation of WSP systems on MI-6 test rig. Vehicle System Dynamics, 44(sup1):843-852, 2006.

Maksym Spiryagin, Colin Cole, Y Sun, Mitchell Mcclanachan, Valentyn Spiryagin, and Tim McSweeney. Design and Simulation of Rail Vehicles. 05 2014. ISBN 9781466575660.

Mirko Tischer and Dietmar Widmann. Model based testing and hardware-in-the-loop simulation of embedded canopen control devices. pages 06/19-06/28. iCC, CAN in Automation, 2012. 